\title{
CHEBYSHEV-VANDERMONDE SYSTEMS
}

\author{
LOTHAR REICHEL AND GERHARD OPFER
}

Dedicated to the memory of Lothar Collatz

ABstract. A Chebyshev-Vandermonde matrix

$$
V=\left[p_{j}\left(z_{k}\right)\right]_{j, k=0}^{n} \in \mathbb{C}^{(n+1) \times(n+1)}
$$

is obtained by replacing the monomial entries of a Vandermonde matrix by Chebyshev polynomials $p_{j}$ for an ellipse. The ellipse is also allowed to be a disk or an interval. We present a progressive scheme for allocating distinct nodes $z_{k}$ on the boundary of the ellipse such that the Chebyshev-Vandermonde matrices obtained are reasonably well-conditioned. Fast progressive algorithms for the solution of the Chebyshev-Vandermonde systems are described. These algorithms are closely related to methods recently presented by Higham. We show that the node allocation is such that the solution computed by the progressive algorithms is fairly insensitive to perturbations in the right-hand side vector. Computed examples illustrate the numerical behavior of the schemes. Our analysis can also be used to bound the condition number of the polynomial interpolation operator defined by Newton's interpolation formula. This extends earlier results of Fischer and the first author.

\section{INTRODUCTION}

Let $E_{\rho}$, for some $\rho \in[0,1]$, be the closed ellipse with boundary curve

$$
\partial E_{\rho}:=\left\{e^{i t}+\rho e^{-i t}: 0 \leq t \leq 2 \pi\right\}, \quad i:=\sqrt{-1} .
$$

Define the polynomials in $z=w+\rho w^{-1}$,

$$
\left\{\begin{array}{l}
p_{0}(z):=1, \\
p_{j}(z):=w^{j}+(\rho / w)^{j} ; \quad j=1,2,3, \ldots .
\end{array}\right.
$$

It can easily be shown (see, e.g., Smirnov and Lebedev [17, Chapter 5]) that the $p_{j}$ are Chebyshev polynomials for $E_{\rho}$ with leading coefficient one, i.e., among all monic polynomials of degree $j, p_{j}$ is the unique polynomial of minimum uniform norm on $E_{\rho}$.

Received September 26, 1988; revised May 16, 1989, November 6, 1989, April 18, 1990.

1980 Mathematics Subject Classification (1985 Revision). Primary 65F05, 65F35, 65D05, $65 \mathrm{D} 32$.

Key words and phrases. Vandermonde-like matrix, ordering of nodes, numerical conditioning, progressive algorithm, Newton interpolation formula.

The first author's research was supported in part by the IBM Bergen Scientific Centre and by NSF under Grant DMS-8704196. 
Example 1.1. Let $\rho:=0$. Then $E_{0}$ is the closed unit disk, and the $p_{j}$ are the monomials $p_{j}(z)=z^{j}, j \geq 0$.

Example 1.2. Let $\rho:=1$. Then $\partial E_{1}=E_{1}$ is the interval $[-2,2]$, and the $p_{j}$ are the 'ordinary' Chebyshev polynomials on $[-2,2]$, i.e., $p_{j}(z)=$ $2 \cos (j \arccos (z / 2)), j \geq 1$.

Chebyshev-Vandermonde matrices (henceforth abbreviated $C V$ matrices) $V=V_{\rho}=\left[v_{j k}\right]_{j, k=0}^{n}, v_{j k}:=p_{j}\left(z_{k}\right)$, arise naturally in polynomial interpolation problems when the basis (1.2) is used for $\Pi_{n}$, the set of polynomials of degree at most $n$. Let $\left(z_{k}, f_{k}\right) \in \mathbb{C}^{2}, 0 \leq k \leq n$, be the given data, where the nodes $z_{k}$ are assumed to be pairwise distinct. The computation of an interpolating polynomial $q_{n} \in \Pi_{n}$ such that $q_{n}\left(z_{k}\right)=f_{k}, 0 \leq k \leq n$, in the form

$$
q_{n}(z)=\sum_{j=0}^{n} a_{j} p_{j}(z)
$$

can be accomplished by solving a dual $C V$ system, namely

$$
V_{\rho}^{\mathrm{T}} \mathbf{a}=\mathbf{f},
$$

where $\mathbf{a}:=\left(a_{0}, a_{1}, \ldots, a_{n}\right)^{\mathrm{T}}$ and $\mathbf{f}:=\left(f_{0}, f_{1}, \ldots, f_{n}\right)^{\mathrm{T}}$. Primal $C V$ systems

$$
V_{\rho} \mathbf{a}=\mathbf{g}, \quad \mathbf{g}:=\left(g_{0}, g_{1}, \ldots, g_{n}\right)^{\mathrm{T}},
$$

arise in the computation of weights of interpolatory quadrature rules with nodes $z_{k}$ when the polynomial basis (1.2) is used. We note that the CV matrix $V_{\rho}$ simplifies to an 'ordinary' Vandermonde matrix when $\rho=0$ in (1.2) (cf. Example 1.1).

Our interest in the basis (1.2) and in fast solution methods for the linear systems of equations (1.4) and (1.5) stems from our ability to bound the growth with $n$ of the condition numbers of the $\mathrm{CV}$ matrices $V_{\rho}$ for certain progressively allocated nodes on $\partial E_{\rho}$. Introduce the condition number

$$
\kappa_{p}\left(V_{\rho}\right):=\left\|V_{\rho}\right\|_{p}\left\|V_{\rho}^{-1}\right\|_{p},
$$

where \|\|$_{p}$ denotes the usual matrix $p$-norm on $\mathbb{C}^{(n+1) \times(n+1)}[12$, p. 56]. We show in $\S 3$ that for our progressively determined nodes the condition number $\kappa_{\infty}\left(V_{\rho}\right)$ grows at most polynomially ${ }^{1}$ with $n$ for any (fixed) $\rho \in[0,1)$. For $\rho=1$, the condition number $\kappa_{\infty}\left(V_{1}\right)$ grows at most like $n^{O(\log n)}$. The latter

\footnotetext{
${ }^{1}$ This bound has for $\rho=0$ recently been improved by A. Córdova, W. Gautschi, and S. Ruscheweyh (see Addendum at the end of this paper).
} 
bound should be compared with recent results by Gautschi and Inglese [8], who show that for real nodes $z_{k}$ the condition number $\kappa_{\infty}\left(V_{0}\right)$ generally grows at least like $O\left(2^{n / 2}\right)$ with $n$. Related results and examples can also be found in [9, $10,11]$. These results indicate that unless special care is taken when allocating the nodes $z_{k}$, the condition numbers of Vandermonde and CV matrices in general grow exponentially with $n$.

The numerical solution of the linear systems of equations (1.4) and (1.5) has received considerable attention when $V_{\rho}$ is an 'ordinary' Vandermonde matrix, i.e., when $\rho=0$. Then the systems (1.4) and (1.5) can be solved in $O\left(n^{2}\right)$ arithmetic operations by methods of Björck and Pereyra [1] and Tang and Golub [18]. This operation count compares favorably with the $O\left(n^{3}\right)$ arithmetic operations required for the solution of (1.4) or (1.5) by Gaussian elimination. Recently Higham [13, 14] presented (nonprogressive) algorithms for the solution of Vandermonde-like linear systems of equations involving polynomials that satisfy a three-term recurrence relation. These algorithms are obtained by modifying the nonprogressive algorithms for 'ordinary' Vandermonde systems in [1].

Our scheme for progressively determining nodes $z_{k}$ makes it attractive to use progressive algorithms for the solution of the CV systems (1.4) and (1.5); i.e., the solution of (1.4) and (1.5) for $n=m+1$ is computed by modifying the solution obtained for $n=m$. Progressive algorithms allow us to conveniently solve (1.4) and (1.5) for increasing values of $n$ until the computed interpolation polynomial $q_{n}$ approximates a given function sufficiently accurately, or until the determined quadrature rule yields a small enough integration error. In $\S 2$ we modify progressive algorithms of Björck and Pereyra [1] in order to obtain progressive $\mathrm{CV}$ solvers that require $O\left(n^{2}\right)$ arithmetic operations and $O(n)$ storage locations for the solution of (1.4) and (1.5) for any $\rho \in[0,1]$ and $V_{\rho} \in \mathbb{C}^{(n+1) \times(n+1)}$. If $\rho=0$, then our progressive $\mathrm{CV}$ solvers simplify to the progressive Vandermonde solvers in [1].

The error propagation of $\mathrm{CV}$ solvers does not only depend on the condition number $\kappa_{p}\left(V_{\rho}\right)$, but also on the ordering of the nodes $z_{k}$. For instance, let $\rho=0$ (unit disk case, cf. Example 1.1) and let the nodes $z_{k}, 0 \leq k \leq n$, be some enumeration of the $n+1$ roots of unity $\{\exp (2 \pi i k /(n+1))\}_{k=0}^{n}$. Then the Vandermonde matrix $V_{0}$ is a scalar multiple of an orthogonal matrix, and therefore $\kappa_{2}\left(V_{0}\right)=1$. However, if $z_{k}=\exp (2 \pi i k /(n+1))$, the error in the solution due to propagated roundoff errors grows rapidly with $n$ (see Figures 4.2.2 and 4.2.4 of $\$ 4$ ). On the other hand, the CV solvers yield a fairly small amplification of roundoff errors if the $z_{k}$ are ordered in such a way that the nodes in each subset $\left\{z_{k}\right\}_{k=0}^{l}, 0 \leq l \leq n$, are 'approximately uniformly distributed' on the unit circle (see Examples $4.2-4.3$ of $\S 4$ ). Such an ordering is given by

$$
z_{k}:=\exp \left(2 \pi i c_{k}\right), \quad 0 \leq k \leq n,
$$


where $\left\{c_{k}\right\}_{k=0}^{\infty}$ is the van der Corput sequence defined as follows. Let the nonnegative integer $k$ have the binary representation

$$
k=\sum_{j=0}^{\infty} k_{j} 2^{j}, \quad k_{j} \in\{0,1\} .
$$

Then $c_{k}$ is given by

$$
c_{k}:=\sum_{j=0}^{\infty} k_{j} 2^{-j-1} .
$$

Table 1.1 shows some values of $c_{k}$ and $\arg \left(z_{k}\right)$ defined by (1.7) and (1.8).

TABLE 1.1

The van der Corput sequence

\begin{tabular}{c|cccccccc}
\hline$k$ & 0 & 1 & 2 & 3 & 4 & 5 & 6 & 7 \\
\hline$c_{k}$ & 0 & $\frac{4}{8}$ & $\frac{2}{8}$ & $\frac{6}{8}$ & $\frac{1}{8}$ & $\frac{5}{8}$ & $\frac{3}{8}$ & $\frac{7}{8}$ \\
\hline $2 \pi c_{k}$ & 0 & $\pi$ & $\frac{\pi}{2}$ & $\frac{3 \pi}{2}$ & $\frac{\pi}{4}$ & $\frac{5 \pi}{4}$ & $\frac{3 \pi}{4}$ & $\frac{7 \pi}{4}$ \\
\hline
\end{tabular}

Properties of the van der Corput sequence are discussed by, e.g., Hlawka [15, p. 93], and properties of the nodes (1.7) are considered in $\S 3$, as well as in [6, 7]. In $\S 3$ we use the van der Corput sequence to allocate nodes $z_{k}$ on $\partial E_{\rho}$ as follows. If $0 \leq \rho<1$, then we let $\alpha \in \mathbb{R}$ be an arbitrary but fixed constant and define

$$
z_{k}:=\exp \left(2 \pi i\left(c_{k}+\alpha\right)\right)+\rho \exp \left(-2 \pi i\left(c_{k}+\alpha\right)\right) \in \partial E_{\rho}
$$

$$
k=0,1,2, \ldots
$$

If instead $\rho=1$, then we define nodes on $[-2,2]$ by

$$
\left\{\begin{array}{l}
z_{0}:=-2, \\
z_{k}:=2 \cos \left(\pi c_{k-1}\right), \quad k=1,2,3, \ldots .
\end{array}\right.
$$

Example 1.3. Let $l \geq 0$ be an arbitrary integer. Assume first that $\rho=0$. Then the set of nodes $\left\{z_{k}\right\}_{k=0}^{2^{l}-1}$ defined by $(1.9)$ is a set of equidistant points on the unit circle. More generally, for any fixed $\rho \in[0,1)$, the set of nodes $\left\{z_{k}\right\}_{k=0}^{2^{l}-1}$ is a set of so-called Fejér points on $\partial E_{\rho}$. Fejér points are defined in, e.g., [17, Chapter 1] and [6,7].

Example 1.4. Let $l \geq 0$ be an arbitrary integer. Then the set of nodes $\left\{z_{k}\right\}_{k=0}^{2^{l}}$ defined by $(1.10)$ is the set of extreme points of the Chebyshev polynomial $p_{2^{l}}(x):=2 \cos \left(2^{l} \arccos (x / 2)\right)$ for the interval $[-2,2]$.

In $\S 3$ we present bounds for the propagated error due to errors in the righthand side for the CV solvers when the matrices $V_{\rho}$ are defined by the nodes 
(1.9) or (1.10). These bounds grow slower than exponentially with $n$. If we would use $c_{k}:=k / n$ in (1.9) and (1.10), then the error in the computed solution would grow exponentially with $n$. This is illustrated by computed examples in $\S 4$.

We finally remark that for a fixed value of $n$, and the nodes (1.9) or (1.10), the solution of (1.4) can be computed by the fast Fourier transform method in $O(n \log n)$ arithmetic operations (see, e.g., Ellacott [4] for a discussion on the use of nodes (1.9)). However, it is difficult to make this approach efficient in a progressive algorithm.

\section{PRogressive Algorithms For CV systems}

This section describes progressive algorithms for the solution of linear systems (1.4) and (1.5), and introduces notation to be used in the analysis of $\S 3$. Our derivation of the algorithms follows closely the derivation by Björck and Pereyra [1] of progressive algorithms for the solution of 'ordinary' Vandermonde systems.

Let $\left(z_{k}, f_{k}\right) \in \mathbb{C}^{2}, 0 \leq k \leq n$, be given data with pairwise distinct nodes $z_{k}$. We wish to compute the coefficients $a_{j}^{(n)}$ of the polynomial

$$
q_{n}(z)=\sum_{j=0}^{n} a_{j}^{(n)} p_{j}(z),
$$

which is uniquely determined by $q_{n}\left(z_{k}\right)=f_{k}, 0 \leq k \leq n$. Following [1], we first express $q_{n}$ in Newton form

$$
q_{n}(z)=: \sum_{j=0}^{n} c_{n-j}^{(n)} \prod_{k=0}^{j-1}\left(z-z_{k}\right)=q_{n-1}(z)+c_{0}^{(n)} \prod_{k=0}^{n-1}\left(z-z_{k}\right),
$$

where empty products are understood to have value one. Assume that the coefficients $a_{j}^{(n-1)}$ of $q_{n-1}$ are already known, and write the product on the right in formula (2.2) as a linear combination of the polynomials $p_{j}$, i.e.,

$$
\sum_{j=0}^{n} b_{j}^{(n)} p_{j}(z):=\prod_{k=0}^{n-1}\left(z-z_{k}\right)
$$

In order to determine the coefficients $b_{j}^{(n)}$, we write (2.3) in the form

$$
\sum_{j=0}^{n} b_{j}^{(n)} p_{j}(z)=\left(z-z_{n-1}\right) \prod_{j=0}^{n-2}\left(z-z_{j}\right)=\left(z-z_{n-1}\right) \sum_{j=0}^{n-1} b_{j}^{(n-1)} p_{j}(z),
$$

and assume that the coefficients $b_{j}^{(n-1)}$ are already known. The $b_{j}^{(n)}$ can now be determined by substituting (1.2) and $z=w+\rho w^{-1}$ into (2.4), and comparing coefficients of equal nonnegative powers of $w$ on the left and right. We obtain 
in this manner, for $n \geq 2$,

$$
\left\{\begin{array}{l}
b_{0}^{(n)}=-z_{n-1} b_{0}^{(n-1)}+2 \rho b_{1}^{(n-1)} \\
b_{j}^{(n)}=b_{j-1}^{(n-1)}-z_{n-1} b_{j}^{(n-1)}+\rho b_{j+1}^{(n-1)}, \quad 1 \leq j \leq n-2, \\
b_{n-1}^{(n)}=b_{n-2}^{(n-1)}-z_{n-1} b_{n-1}^{(n-1)} \\
b_{n}^{(n)}=1 .
\end{array}\right.
$$

Finally, substituting (2.1) and (2.3) into (2.2) and comparing coefficients of the $p_{j}$ yields expressions for the $a_{j}^{(n)}$ in terms of the coefficients $a_{j}^{(n-1)}$ :

$$
\left\{\begin{array}{l}
a_{0}^{(0)}=f_{0}, \\
a_{j}^{(n)}=a_{j}^{(n-1)}+c_{0}^{(n)} b_{j}^{(n)}, \quad j=0,1, \ldots, n-1, \\
a_{n}^{(n)}=c_{0}^{(n)} b_{n}^{(n)} .
\end{array}\right.
$$

Combining the above formulas gives rise to the following algorithm.

Algorithm 1. Progressive algorithm for the solution of dual CV systems.

Data: $\rho ;\left(z_{n}, f_{n}\right), n=0,1,2, \ldots$

$c_{0}^{(0)}:=f_{0} ; a_{0}^{(0)}:=f_{0}$;

for $n:=1,2,3, \ldots$ until no more nodes do

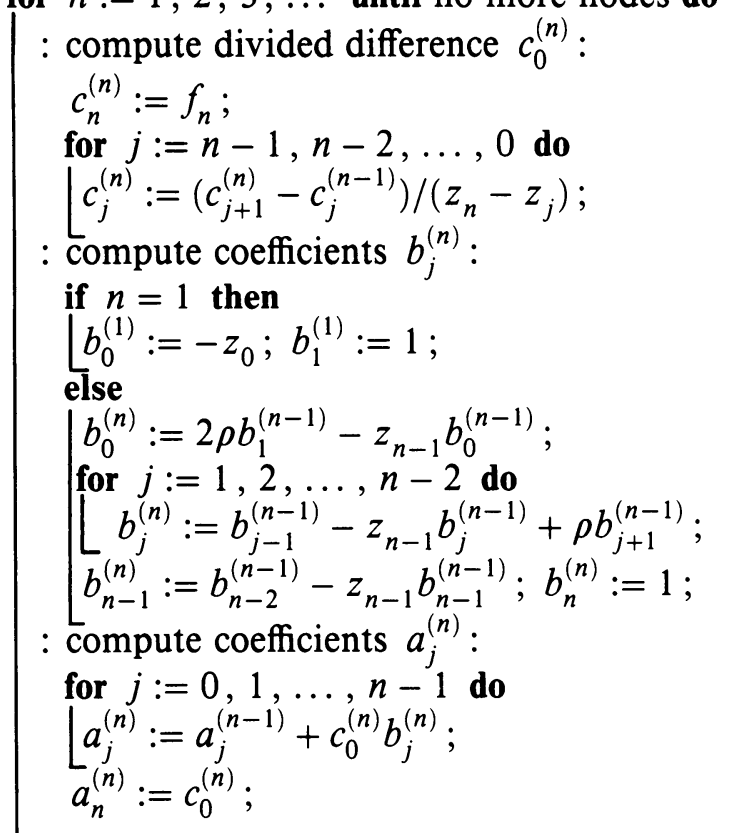

We remark that the nodes in Algorithm 1 are arbitrary pairwise distinct nodes. The value of $\rho$ determines the polynomial basis. Two FORTRAN subroutines for Algorithm 1 are listed in [16]: one for complex nodes and $0 \leq \rho<1$, using complex arithmetic, and one for the important special case of real nodes and $\rho=1$, using real arithmetic only. The codes are available from the authors. The subroutines require $O(n)$ storage locations in order to compute the coefficients 
$\left\{a_{j}^{(n)}\right\}_{j=0}^{n}$ of (2.1). The operation count for computing these coefficients by the code for real nodes and $\rho=1$ is $\frac{3}{2} n^{2}+O(n)$ multiplications or divisions and $\frac{5}{2} n^{2}+O(n)$ additions or subtractions. If we compute the coefficients $\left\{a_{j}^{(n)}\right\}_{j=0}^{n}$ by the code for complex nodes and $0 \leq \rho<1$, and convert complex arithmetic operations into real ones, then $10 n^{2}+O(n)$ real multiplications or divisions are required. This operation count is based on the observation that one complex multiplication takes three real multiplications, and one complex division takes six real multiplications or divisions.

We now turn to the derivation of a progressive algorithm for the solution of primal CV systems (1.5). Following the approach used in [1] for the derivation of Vandermonde solvers, we first make a matrix interpretation of Algorithm 1. The matrices introduced will be used in the error analysis of $\S 3$.

Let $m$ be an arbitrary integer larger than or equal to $n$. For $0 \leq k<m$, define the matrices

$$
L_{k}:=\left(\begin{array}{c|ccccc}
I_{k+1} & & & 0 & & \\
\hline & 1 & 0 & \ldots & & 0 \\
\mathbf{0} & -1 & 1 & & & \\
& \vdots & \ddots & \ddots & & \vdots \\
& & & -1 & 1 & 0 \\
& 0 & & & -1 & 1
\end{array}\right) \in \mathbb{R}^{(m+1) \times(m+1)}
$$

and

$$
\begin{aligned}
D_{k} & :=\operatorname{diag}\left[1,1, \ldots, 1,\left(z_{k+1}-z_{0}\right)^{-1},\left(z_{k+2}-z_{1}\right)^{-1}, \ldots,\left(z_{m}-z_{m-k-1}\right)^{-1}\right] \\
& \in \mathbb{C}^{(m+1) \times(m+1)},
\end{aligned}
$$

where $I_{j}$ denotes the identity matrix of order $j$. Introduce the coordinate vectors $\mathbf{e}_{j}$, i.e., $\mathbf{e}_{j}$ is the $(j+1)$ st column of $I_{m+1}$. Then the divided difference $c_{0}^{(n)}$ in formula $(2.2)$ can be written as

$$
c_{0}^{(n)}=\mathbf{e}_{n}^{\mathrm{T}} D_{n-1} L_{n-1} D_{n-2} L_{n-2} \cdots D_{0} L_{0} \mathbf{f}, \quad 1 \leq n \leq m .
$$

Introduce the tridiagonal matrices

$$
W_{j}:=\left(\begin{array}{ccccccc}
-z_{j} & 2 \rho & 0 & \cdots & & & 0 \\
1 & -z_{j} & \rho & \ddots & & & \vdots \\
0 & 1 & -z_{j} & \rho & & & \\
\vdots & & 1 & -z_{j} & \ddots & & \\
& & & \ddots & \ddots & & 0 \\
& & & & 1 & -z_{j} & \rho \\
0 & \cdots & & & 0 & 1 & -z_{j}
\end{array}\right) \in \mathbb{C}^{(m+1) \times(m+1)},
$$

$0 \leq j \leq m$, and let

$$
\begin{aligned}
\tilde{\mathbf{b}}^{(n)}:=\left(b_{0}^{(n)}, b_{1}^{(n)}, \ldots, b_{n}^{(n)}, 0,0, \ldots, 0\right)^{\mathrm{T}} \in \mathbb{C}^{m+1}, & & 0 \leq n \leq m, \\
\tilde{\mathbf{a}}^{(n)}:=\left(a_{0}^{(n)}, a_{1}^{(n)}, \ldots, a_{n}^{(n)}, 0,0, \ldots, 0\right)^{\mathrm{T}} \in \mathbb{C}^{m+1}, & & 0 \leq n \leq m,
\end{aligned}
$$


where the $b_{j}^{(n)}$ and $a_{j}^{(n)}$ are defined by (2.3) and (2.1), respectively. Then

$$
\begin{gathered}
\tilde{\mathbf{b}}^{(n)}=W_{n-1} W_{n-2} \cdots W_{0} \mathbf{e}_{0}, \quad 1 \leq n \leq m, \\
\tilde{\mathbf{a}}^{(n)}=\tilde{\mathbf{a}}^{(n-1)}+\tilde{\mathbf{b}}^{(n)} c_{0}^{(n)}=\tilde{\mathbf{a}}^{(n-1)}+S_{n} \mathbf{f}, \quad 0 \leq n \leq m,
\end{gathered}
$$

where

$$
\begin{cases}S_{n}:=W_{n-1} W_{n-2} \cdots W_{0} \mathbf{e}_{0} \mathbf{e}_{n}^{\mathrm{T}} D_{n-1} L_{n-1} D_{n-2} L_{n-2} \cdots & D_{0} L_{0}, \\ S_{0}:=\mathbf{e}_{0} \mathbf{e}_{0}^{\mathrm{T}}, & 1 \leq n \leq m,\end{cases}
$$

and $\tilde{\mathbf{a}}^{(-1)}:=\mathbf{0}$. Hence,

$$
\tilde{\mathbf{a}}^{(n)}=\sum_{j=0}^{n} S_{j} \mathbf{f}, \quad 1 \leq n \leq m .
$$

Let $\widetilde{V}$ denote the $\mathrm{CV}$ matrix of order $m+1$ defined by the node set $\left\{z_{j}\right\}_{j=0}^{m}$ and $\rho \in[0,1]$. It follows from (1.4) and (2.9) that

$$
\tilde{V}^{-\mathrm{T}}=\sum_{j=0}^{m} S_{j}
$$

and therefore

$$
\tilde{V}^{-1}=\sum_{j=0}^{m} S_{j}^{\mathrm{T}} .
$$

From (2.11), and the fact that the $W_{j}$ commute, we obtain the following algorithm for the solution of (1.5):

Algorithm 2. Progressive algorithm for the solution of primal CV systems.

Data: $\rho ;\left(z_{n}, f_{n}\right), n=0,1,2, \ldots$

$f_{0}^{(0)}:=f_{0} ; a_{0}^{(0)}:=f_{0} ; u_{0}^{(0)}:=1$;

for $n:=1,2,3, \ldots$ until no more nodes do

: compute $f_{n}^{(n)}:=\mathbf{e}_{0}^{\mathrm{T}} W_{0}^{\mathrm{T}} W_{1}^{\mathrm{T}} \cdots W_{n-1}^{\mathrm{T}} \mathbf{f}$ :

$f_{0}^{(n)}:=f_{n}$

for $k:=0,1, \ldots, n-2$ do

$f_{k+1}^{(n)}:=f_{k}^{(n)}-z_{k} f_{k}^{(n-1)}+\rho f_{k}^{(n-2)}$;

if $k=n-2$ then $f_{k+1}^{(n)}:=f_{k+1}^{(n)}+\rho f_{k}^{(n-2)}$;

$f_{n}^{(n)}:=f_{n-1}^{(n)}-z_{n-1} f_{n-1}^{(n-1)}$;

: compute $\mathbf{u}^{(n)}:=\left(1 / w_{0}^{(n)}, 1 / w_{1}^{(n)}, \ldots, 1 / w_{n}^{(n)}\right)^{\mathrm{T}}$, where

$\mathbf{w}^{(n)}=\left(w_{0}^{(n)}, w_{1}^{(n)}, \ldots, w_{n}^{(n)}\right)^{\mathrm{T}}:=L_{0}^{\mathrm{T}} D_{0} L_{1}^{\mathrm{T}} D_{1} \cdots L_{n-1}^{\mathrm{T}} D_{n-1} \mathbf{e}_{n}:$

$u_{n}^{(n)}:=1$

for $k:=0,1, \ldots, n-1$ do

$\left[\begin{array}{l}u_{k}^{(n)}:=\left(z_{k}-z_{n}\right) u_{k}^{(n-1)} \\ u_{n}^{(n)}:=\left(z_{n}-z_{k}\right) u_{n}^{(n)}\end{array}\right.$ 


$$
\left[\begin{array}{l}
: \text { compute } \mathbf{a}^{(n)}:=\mathbf{a}^{(n-1)}+\mathbf{w}^{(n)} f_{n}^{(n)}: \\
\text { for } k:=0,1, \ldots, n-1 \text { do } \\
{\left[\begin{array}{c}
(n) \\
a_{k}^{(n)}:=a_{k}^{(n-1)}+f_{n}^{(n)} / u_{k}^{(n)}
\end{array}\right.} \\
a_{n}^{(n)}:=f_{n}^{(n)} / u_{n}^{(n)}
\end{array}\right.
$$

Two FORTRAN subroutines for Algorithm 2 are listed in [16]: one for complex nodes and $0 \leq \rho<1$, and one for real nodes and $\rho=1$. The codes are available from the authors. Similarly as for Algorithm 1, the nodes for Algorithm 2 are assumed to be pairwise distinct but otherwise arbitrary, and $\rho$ determines the polynomial basis (1.2).

\section{CONDITION NUMBER BOUNDS}

In this section we assume that the nodes $z_{k}$ are given by (1.9) or (1.10). We derive bounds for the rate of growth with $n$ of the condition numbers (1.6) of the $\mathrm{CV}$ matrices $V_{\rho}$ of order $n+1$. Also, we present bounds for propagated errors due to errors in the right-hand side vectors in (1.4) and (1.5). These bounds are derived by bounding the quantities computed by Algorithms 1 and 2; i.e., in order to bound $V_{\rho}^{-1}$, we bound the mapping from the right-hand side vector in (1.4) to the divided differences $c_{n-j}^{(n)}$ in (2.2), and the mapping from the divided differences to the solution vector $\mathbf{a}$. Our analysis extends previous results in [7] on bounds for the condition number for the Newton interpolation formula. This application will be discussed in Remark 3.1 below. by

Introduce the mappings $M_{1}: \mathbb{C}^{n+1} \rightarrow \mathbb{C}^{n+1}$ and $M_{2}: \mathbb{C}^{n+1} \rightarrow \mathbb{C}^{n+1}$ defined

$$
\begin{aligned}
M_{1} \mathbf{f}:=\mathbf{c} & =\left(c_{0}^{(n)}, c_{1}^{(n)}, \ldots, c_{n}^{(n)}\right)^{\mathrm{T}}, \\
M_{2} \mathbf{c} & :=\mathbf{a}=\left(a_{0}^{(n)}, a_{1}^{(n)}, \ldots, a_{n}^{(n)}\right)^{\mathrm{T}},
\end{aligned}
$$

where the $c_{j}^{(n)}$ are the divided differences of the Newton form (2.2), and a solves (1.4). By using the orthogonality of the $p_{. j}$ with respect to one of the inner products

$$
\begin{aligned}
& \left\langle\Psi_{1}, \Psi_{2}\right\rangle_{\rho}:=\frac{1}{2 \pi} \int_{E_{\rho}} \overline{\Psi_{1}(z)} \Psi_{2}(z)\left|z^{2}-4 \rho\right|^{-1 / 2}|d z|, \quad 0 \leq \rho<1, \\
& \left\langle\Psi_{1}, \Psi_{2}\right\rangle_{\rho}:=\frac{1}{\pi} \int_{-2}^{2} \Psi_{1}(x) \Psi_{2}(x)\left|x^{2}-4\right|^{-1 / 2} d x, \quad \rho=1,
\end{aligned}
$$

we can bound the mapping $M_{2}$ in a fairly straightforward manner. The derivation of a bound for $M_{1}$ requires more work and will be discussed first. Most oî the proofs are just outlined; details can be found in [16].

Equip $\mathbb{C}^{n+1}$ with the uniform norm,

$$
\|\mathbf{v}\|_{\infty}:=\max _{0 \leq j \leq n}\left|v_{j}\right|, \quad \mathbf{v}=\left(v_{0}, v_{1}, \ldots, v_{n}\right)^{\mathrm{T}},
$$


and let $\left\|M_{j}\right\|_{\infty}$ denote the induced operator norm. In order to bound $\left\|M_{1}\right\|_{\infty}$, we first note that the divided differences $c_{j}^{(n)}$ can be written as

$$
c_{n-j}^{(n)}=\sum_{k=0}^{j} \frac{f_{k}}{\prod_{l=0, l \neq k}^{j}\left(z_{k}-z_{l}\right)}, \quad 0 \leq j \leq n,
$$

(see, e.g., Davis $[2, \S 2.6])$. A lower bound for the products in (3.4) was derived in [7] for $z_{k}$ given by (1.9) and $0 \leq \rho<1$. This bound is used in the proof of the following theorem.

Theorem 3.1. Let the nodes $z_{k}, 0 \leq k \leq n$, be defined by (1.9) for an arbitrary constant $\alpha \in \mathbb{R}$, and let $0 \leq \rho<1$. Then there are nonnegative constants $d_{1}, d_{2}$ depending on $\rho$, but independent of $n$, such that

$$
\left\|M_{1}\right\|_{\infty} \leq d_{1}(n+1)^{d_{2}}, \quad n \geq 1 .
$$

Proof. By (3.4),

$$
\left\|M_{1}\right\|_{\infty}=\max _{\|\|_{\infty}=1}\|\mathbf{c}\|_{\infty} \leq \max _{0 \leq j \leq n} \sum_{k=0}^{j} \sum_{\substack{l=0 \\ l \neq k}}^{j}\left|z_{k}-z_{l}\right|^{-1} .
$$

The right-hand side of (3.6) can be bounded by applying Lemma 2.5 of [7], and (3.5) follows.

In a sequence of lemmas we now present some auxiliary results that we use to bound $\left\|M_{1}\right\|_{\infty}$ for $\rho=1$ and the nodes (1.10):

Lemma 3.1. Let the nodes $z_{k}$ be defined by (1.10). Then

$$
\prod_{\substack{j=0 \\ j \neq k}}^{2^{l}}\left|z_{k}-z_{j}\right| \geq 2^{l+1}, \quad 0 \leq k \leq 2^{l}
$$

for any integer $l \geq 0$.

Proof. Let $U_{n}(x):=\sin ((n+1) \theta) / \sin \theta$, where $x=\cos \theta$, denote Chebyshev polynomials of the second kind. Then

$$
\prod_{j=0}^{2^{l}}\left(z-z_{j}\right)=\left(z^{2}-4\right) U_{2^{l}-1}\left(\frac{z}{2}\right), \quad-2 \leq z \leq 2 .
$$

By evaluating, and then estimating, the derivative of (3.8) at $z=z_{k}$ we obtain (3.7).

Let the nodes $z_{k}$ be defined by (1.10). Then Lemma 3.1 yields

$$
\prod_{\substack{j=0 \\ j \neq k}}^{n}\left|z_{k}-z_{j}\right|^{-1} \leq 2^{-l-1} \prod_{j=n+1}^{2^{l}}\left|z_{k}-z_{j}\right|, \quad 0 \leq k \leq n,
$$

where as usual the empty product is defined to have value one. The lemma below bounds the right-hand side of (3.9). 
Lemma 3.2. Let the nodes $z_{j}$ be defined by (1.10) and assume that $1 \leq n<2^{l}$. Then

$$
\prod_{m=n+1}^{2^{l}}\left|z_{k}-z_{m}\right| \leq 2^{l(l+1)} .
$$

Proof. The bound is obtained by partitioning the product (3.10) into subproducts, each of which contains factors $\left|z_{k}-z_{m}\right|$ with nodes $z_{m}$ that are distributed like the first $2^{l}$ points determined by $(1.10)$ for some integer $l \geq 0$. Such a partitioning is described by [6, Lemma 2.4]. Each subproduct can be bounded, and (3.10) is obtained.

We are now in a position to show a result for the nodes (1.10) analogous to Theorem 3.1.

Theorem 3.2. Let the nodes $z_{k}, 0 \leq k \leq n$, be defined by (1.10), and let $\rho=1$. Then

$$
\left\|M_{1}\right\|_{\infty} \leq 2 n^{3+\log _{2} n}, \quad n \geq 1 .
$$

Proof. From (3.6) we obtain

$$
\begin{aligned}
\left\|M_{1}\right\|_{\infty} & \leq \max _{0 \leq j \leq n} \sum_{k=0}^{j} \sum_{\substack{m=0 \\
m \neq k}}^{j}\left|z_{k}-z_{m}\right|^{-1} \\
& =\max \left\{1, \max _{1 \leq j \leq n} \sum_{\substack { k=0 \\
\begin{subarray}{c}{m=0 \\
m \neq k{ k = 0 \\
\begin{subarray} { c } { m = 0 \\
m \neq k } }\end{subarray}}^{j}\left|z_{k}-z_{m}\right|^{-1}\right\} .
\end{aligned}
$$

Let $l \geq 0$ be the unique integer such that $n<2^{l} \leq 2 n$. An application of (3.7) and (3.10) yields

$$
\begin{aligned}
& \max _{1 \leq j \leq n} \sum_{k=0}^{j} \prod_{\substack{m=0 \\
m \neq k}}^{j}\left|z_{k}-z_{m}\right|^{-1}=\max _{1 \leq j \leq n} \sum_{-k=0}^{j} \frac{\prod_{m=j+1}^{2^{l}}\left|z_{k}-z_{m}\right|}{\prod_{m=0, m \neq k}^{2^{l}}\left|z_{k}-z_{m}\right|} \\
& \quad \leq \max _{1 \leq j \leq n} 2^{-l-1} \sum_{k=0}^{j} \prod_{m=j+1}^{2^{l}}\left|z_{k}-z_{m}\right|^{-1} \leq \max _{1 \leq j \leq n} 2^{-l-1} \sum_{k=0}^{j} 2^{l(l+1)} \\
& \quad=\frac{1}{2}(n+1) 2^{l \cdot l} \leq \frac{1}{2}(n+1)(2 n)^{l} \leq(n+1) n^{2+\log _{2} n}, \quad n \geq 1 .
\end{aligned}
$$

Substitution of this inequality into (3.12) shows (3.11).

Remark 3.1. In [7] the stability of the Newton interpolation formula is discussed for interpolation at nodes on a smooth Jordan curve. The nodes considered are Fejér points ordered by the van der Corput sequence, such as the nodes (1.9). A mapping $T$ is defined that maps the vector $\mathbf{f}:=\left(f_{0}, f_{1}, \ldots, f_{n}\right)^{\mathrm{T}}$ to the polynomial $q_{n} \in \Pi_{n}$ in Newton form (cf. (2.2)). The range and domain of $T$ 
are equipped with the uniform norm, and it is shown in [7, Theorem 2.6] that $\lim _{n \rightarrow \infty} \operatorname{cond}(T)^{1 / n}=1$, where $\operatorname{cond}(T)$ denotes the condition number of $T$. By using Theorem 3.2, this equality can also be shown when interpolation is carried out at the nodes (1.10). This result follows by substituting (3.11) into the proof of [7, Theorem 2.6].

We now derive a bound for $\left\|M_{2}\right\|_{\infty}$. This is achieved by first bounding the products $\prod_{k=0}^{j-1}\left(z-z_{k}\right)$ in the Newton form (2.2), and then using the orthogonality of the $p_{j}$ with respect to one of the inner products (3.3).

Lemma 3.3. Let $0 \leq \rho<1$ and let the nodes $z_{k}, 0 \leq k<r$, be defined by (1.9). Then

$$
\prod_{k=0}^{r-1}\left|z-z_{k}\right| \leq 4 r^{2}, \quad r \geq 1, \quad z \in \partial E_{\rho} .
$$

If, instead, the nodes $z_{k}, 0 \leq k \leq r$, are defined by (1.10), then

$$
\prod_{k=0}^{r}\left|z-z_{k}\right| \leq 4 r^{\log _{2} 2 r}, \quad r \geq 1, \quad z \in[-2,2] .
$$

Proof. The product (3.13) is partitioned into subproducts, each of which contains $2^{l}$ nodes $z_{k}$ that are distributed roughly like the first $2^{l}$ nodes (1.9). Such a partitioning is described by [6, Lemma 2.3]. These subproducts can be bounded, and (3.13) is obtained. The proof of (3.14) is analogous.

We are now in a position to bound the mapping $M_{2}$. The bounds show that the norm of $M_{2}$ grows fairly slowly with $n$.

Theorem 3.3. Let the nodes $z_{k}, 0 \leq k \leq n$, be defined by (1.9), and let $0 \leq$ $\rho<1$. Then

$$
\left\|M_{2}\right\|_{\infty} \leq 8 n^{3}, \quad n \geq 2 .
$$

If, instead, the nodes $z_{k}, 0 \leq k \leq n$, are given by (1.10), and $\rho=1$, then

$$
\left\|M_{2}\right\|_{\infty} \leq 10 n^{2+\log _{2} n}, \quad n \geq 2 .
$$

Proof. Let the values of $\rho$ in the definition (1.2) of the polynomials $p_{j}$ and in the inner product $\langle\cdot, \cdot\rangle_{\rho}$ given by (3.3) be identical. Then

$$
\left\langle p_{j}, p_{k}\right\rangle_{\rho}=0, \quad k \neq j, \quad 1 \leq\left\langle p_{j}, p_{j}\right\rangle_{\rho} \leq 2, \quad j \geq 0 .
$$

We obtain from (2.1) and the orthogonality of the $p_{j}$ that

$$
a_{j}^{(n)}=\left\langle q_{n}, p_{j}\right\rangle_{\rho} /\left\langle p_{j}, p_{j}\right\rangle_{\rho}, \quad 0 \leq j \leq n .
$$

Now (3.2), (3.17), and (3.18) show that

$$
\left\|M_{2}\right\|_{\infty}=\max _{\|\boldsymbol{c}\|_{\infty}=1}\|\mathbf{a}\|_{\infty} \leq \max _{\|\mathbf{c}\|_{\infty}=1} \max _{0 \leq j \leq n}\left|\left\langle q_{n}, p_{j}\right\rangle_{\rho}\right| .
$$


Substituting

$$
\left|\left\langle q_{n}, p_{j}\right\rangle_{\rho}\right| \leq \max _{z \in \partial E_{\rho}}\left|q_{n}(z)\right| \max _{z \in \partial E_{\rho}}\left|p_{j}(z)\right|, \quad \max _{z \in \partial E_{\rho}}\left|p_{j}(z)\right| \leq 2,
$$

and (2.2) into (3.19) yields

$$
\left\|M_{2}\right\|_{\infty} \leq 2 \max _{\|c\|=1} \max _{z \in \partial E_{\rho}}\left|q_{n}(z)\right| \leq 2 \max _{z \in \partial E_{\rho}} \sum_{j=0}^{n} \prod_{k=0}^{j-1}\left|z-z_{k}\right| .
$$

The right-hand side of (3.21) can be bounded, using Lemma 3.3, and the theorem follows.

We note that formulas similar to (3.15)-(3.16) are valid also if the Chebyshev polynomials $p_{j}$ are replaced by polynomials that belong to some other family of orthogonal polynomials, such as Legendre polynomials. The proof of Theorem 3.3 only requires that an inequality of the form (3.17) is valid.

Theorem 3.4. Let $V_{\rho} \in \mathbb{C}^{(n+1) \times(n+1)}$. Assume that $0 \leq \rho<0$, and let the nodes $z_{k}$ be given by (1.9). Then there are constants $c$ and $d$ depending on $\rho$, but independent of $n$, such that

$$
\kappa_{\infty}\left(V_{\rho}^{\mathrm{T}}\right) \leq c n^{d}, \quad n \geq 1 .
$$

If, instead, $\rho=1$, and the nodes $z_{k}$ are given by (1.10), then

$$
\kappa_{\infty}\left(V_{1}^{\mathrm{T}}\right) \leq 40 n^{5+2 \log _{2} n}, \quad n \geq 1 .
$$

Proof. From the second inequality in (3.20) it follows that $\left\|V_{\rho}^{\mathrm{T}}\right\|_{\infty} \leq 2(n+1)$. The factorization $V_{\rho}^{-\mathrm{T}}=M_{2} M_{1}$ and Theorems 3.1-3.3 yield bounds for $V_{\rho}^{-\mathrm{T}}$, and the theorem follows.

Theorem 3.4 shows that the condition number grows slower than exponentially with $n$ for nodes (1.9) and (1.10). The bounds in Theorems 3.1-3.4 are not sharp, however, and the numerical experiments of $\S 4$ display a quite modest growth of $\kappa_{\infty}\left(V_{\rho}^{\mathrm{T}}\right)$ with $n$. Bounds for $\kappa_{\infty}\left(V_{\rho}\right)=\left\|V_{\rho}\right\|_{\infty}\left\|V_{\rho}^{-1}\right\|_{\infty}$ can be obtained by Theorem 3.4 and the observation that for any matrix $A \in \mathbb{C}^{(n+1) \times(n+1)}$,

$$
\left\|A^{\mathrm{T}}\right\|_{\infty}=\|A\|_{1} \leq(n+1)\|A\|_{\infty} .
$$

We turn to the propagation of errors in the right-hand side vectors in (1.4) and (1.5) by Algorithms 1 and 2. A comparison of (2.5)-(2.8) with (3.1)-(3.2) yields, for $n \geq 1$,

$$
\begin{aligned}
\mathbf{e}_{n}^{\mathrm{T}} D_{n-1} L_{n-1} D_{n-2} L_{n-2} \cdots D_{0} L_{0} & =\mathbf{e}_{0}^{\mathrm{T}} M_{1}, \\
W_{n-1} W_{n-2} \cdots W_{0} \mathbf{e}_{0} & =M_{2} \mathbf{e}_{0}, \\
S_{n} & =M_{2} \mathbf{e}_{0} \mathbf{e}_{0}^{\mathrm{T}} M_{1} .
\end{aligned}
$$

The following theorem shows that the propagated errors in the solution vectors of (1.4) and (1.5), due to perturbations in the right-hand side vectors $f$ and g, grow slower than exponentially with $n$. We remark that for many distributions and orderings of nodes $z_{k}$, the propagated error does, indeed, grow exponentially with $n$ (see the numerical examples of $\S 4$ ). 
Theorem 3.5. Let $0 \leq \rho<0$, and let the nodes $z_{k}$ be given by (1.9). Then there are constants $c_{1}$ and $d_{1}$ independent of $n$ such that

$$
\max \left\{\sum_{j=0}^{n}\left\|S_{j}\right\|_{\infty}, \sum_{j=0}^{n}\left\|S_{j}^{\mathrm{T}}\right\|_{\infty}\right\} \leq c_{1} n^{d_{1}} .
$$

If, instead, $\rho=1$, and the nodes $z_{k}$ are given by (1.10), then there are constants $c_{2}$ and $d_{2}$ independent of $n$ such that

$$
\max \left\{\sum_{j=0}^{n}\left\|S_{j}\right\|_{\infty}, \sum_{j=0}^{n}\left\|S_{j}^{\mathrm{T}}\right\|_{\infty}\right\} \leq c_{2} n^{d_{2} \log _{2}(n)} .
$$

Proof. The proof follows from $\left\|S_{0}\right\|_{\infty}=1$, bounds for $\left\|M_{1}\right\|_{\infty}$ and $\left\|M_{2}\right\|_{\infty}$, and (3.22)-(3.23).

\section{NumericAl EXAMPLES}

The computed examples of this section illustrate the results of $\S 3$. All examples have been computed on an IBM 3090VF computer. Throughout this section the parameter $\alpha$ in (1.9) is set to zero.

Example 4.1. This example shows $\kappa_{2}\left(V_{\rho}\right) \quad\left(=\kappa_{2}\left(V_{\rho}^{\mathrm{T}}\right)\right)$ as a function of $n$ for different values of $\rho$, where $V_{\rho} \in \mathbb{C}^{(n+1) \times(n+1)}$. The condition numbers $\kappa_{2}\left(V_{\rho}\right)$ have been computed in double precision arithmetic, i.e., with 15 significant digits, using the subroutine ZSVDC of LINPACK [3]. Figure 4.1.1 illustrates the oscillating behavior of $n \rightarrow \kappa_{2}\left(V_{0}\right)$, where $V_{0}$ is defined by the nodes (1.9). The condition number $\kappa_{2}\left(V_{0}\right)$ is smallest when $n$ is such that the set of nodes $\left\{z_{k}\right\}_{k=0}^{n}$ can be written as the union of only a few disjoint sets of equidistant nodes. For instance, if $n=2^{l}-1$ for some integer $l \geq 0$, then the $z_{k}$ are the $n$th roots of unity and $V_{0}$ is orthogonal, i.e., $\kappa_{2}\left(V_{0}\right)=1$.

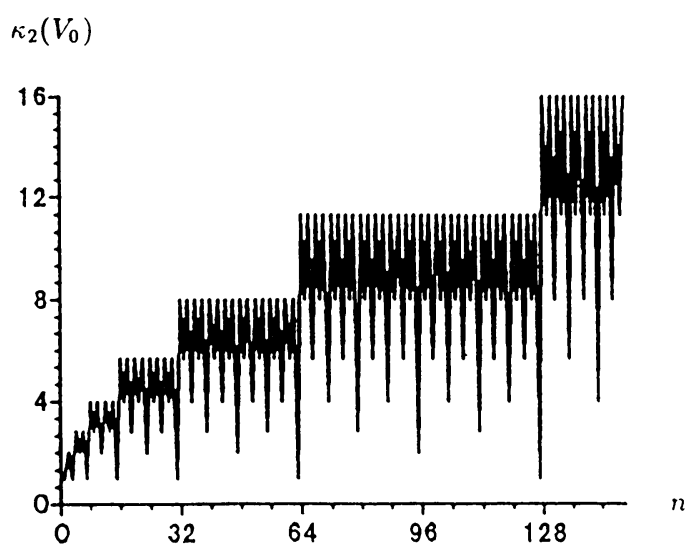

FIGURE 4.1.1

Condition number $\kappa_{2}\left(V_{0}\right)$ as a function of $n$ 


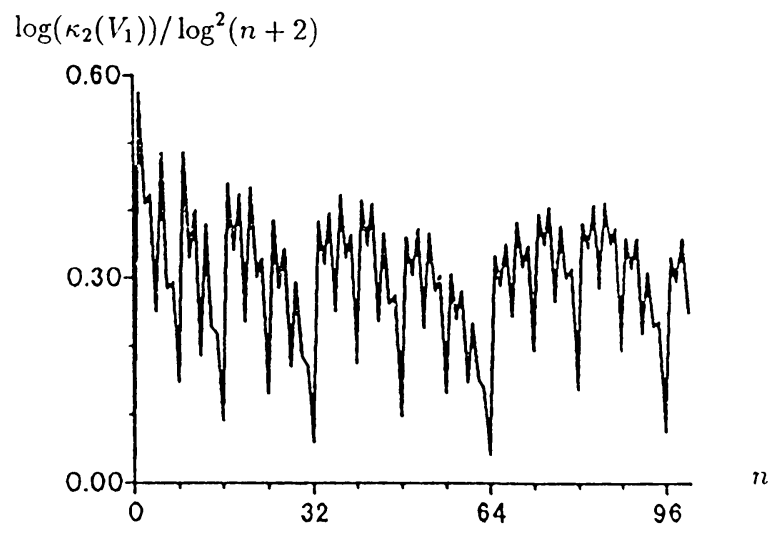

FIGURE 4.1.2

Growth of $\kappa_{2}\left(V_{1}\right)$ with $n$

In Figure 4.1.2 we have chosen $\rho=1$ and the nodes (1.10). This figure suggests that $\kappa_{2}\left(V_{1}\right)<n^{0.45 \log (n)}$ for large $n$, where log denotes the natural logarithm.

The following examples illustrate the propagation of roundoff errors in Algorithms 1 and 2 . Because of the small amplification of roundoff errors when the nodes (1.9) and (1.10) are used, we are able to solve fairly large CV systems (1.4) and (1.5) in single precision arithmetic, i.e., with only six significant digits.

Example 4.2. In this example we solve dual CV systems (1.4) by Algorithm 1 in single precision arithmetic. Let $\mathbf{x} \in \mathbb{C}^{n+1}$ denote the exact solution of (1.4), and let $\mathbf{x}^{*}$ denote the computed solution. We determine the residual error

$$
\mathbf{r}:=V_{\rho}^{\mathrm{T}} \mathbf{x}^{*}-\mathbf{f}
$$

by accumulating sums in double precision arithmetic. The norm $\|\mathbf{r}\|_{\infty}$ is a good estimate for the norm of the error in the solution $\left\|\mathbf{x}^{*}-\mathbf{x}\right\|_{\infty}$ because $V^{\mathrm{T}}$ is quite well-conditioned (see Example 4.1).

Figure 4.2.1 shows $\|\mathbf{r}\|_{\infty}$ when $\rho=0$ and the nodes are defined by (1.9). The real and imaginary parts of the right-hand side $f \in \mathbb{C}^{n+1}$ are uniformly distributed elements in $[0,1]$, computed by the random number generator SURAND of the ESSL program library [5]. The figure shows a slow growth of $\|\mathbf{r}\|_{\infty}$ with $n$.

The computations for Figures 4.2.1 and 4.2.2 differ only in the ordering of the nodes. The matrix $V_{0}^{\mathrm{T}} \in \mathbb{C}^{(n+1) \times(n+1)}$ used for Figure 4.2.2 is defined by the nodes

$$
z_{k}=\exp (2 \pi i k /(n+1)), \quad 0 \leq k \leq n,
$$

for every $n \geq 1$. The nodes (4.2) make $V_{0}^{\mathrm{T}}$ orthogonal for every $n$, but yield severe amplification of roundoff errors, as shown by Figure 4.2.2. The rapid 


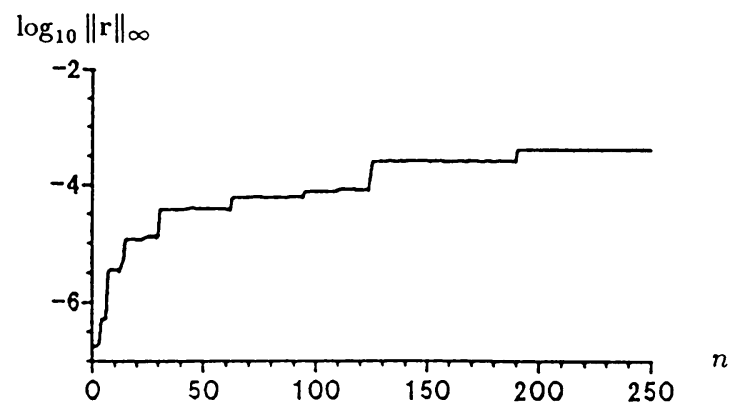

FIgURE 4.2.1

Growth of $\|\mathbf{r}\|_{\infty}$ with $n$ for $\rho=0$ and nodes (1.9)

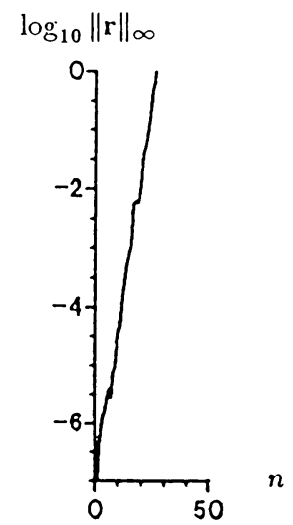

FIGURE 4.2.2

Growth of $\|\mathbf{r}\|_{\infty}$ with $n$ for $\rho=0$ and nodes (4.2)

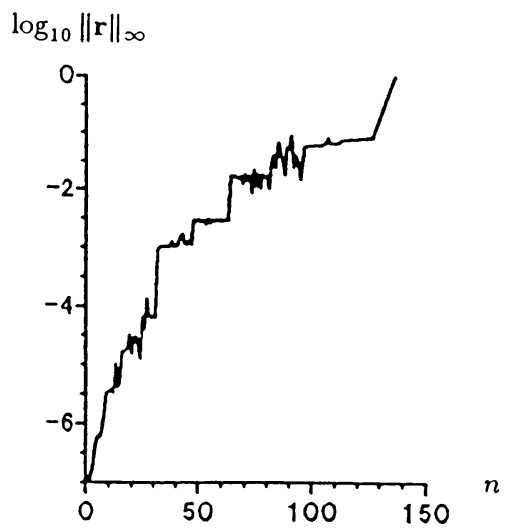

FIGURE 4.2.3

Growth of $\|\mathbf{r}\|_{\infty}$ with $n$ for $\rho=1$ and nodes (1.10) 


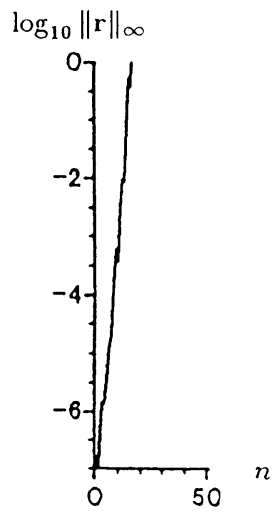

FIGURE 4.2.4

Growth of $\|\mathbf{r}\|_{\infty}$ with $n$ for $\rho=1$ and nodes (4.3)

growth of the propagated error is a result of the ordering of the nodes (4.2), which is unsuitable because it causes many products in the denominators in (3.4) to have tiny magnitude.

Experiments suggest that the graphs of Figures 4.2.1-4.2.2 are quite insensitive to the choice of right-hand side vector $\mathbf{f}$, as well as to the choice of $0 \leq \rho<1$, if $\rho$ is not very close to 1 .

In Figures 4.2 .3 and 4.2.4 we set $\rho=1$ and present graphs analogous to those of Figures 4.2.1-4.2.2. Figure 4.2.3 shows $\|\mathbf{r}\|_{\infty}$ when the nodes $z_{j}$ are given by (1.10) and $\mathbf{f}=\left[f_{j}\right]_{j=0}^{n}$ has elements uniformly distributed in $[0,1]$. The computations for Figure 4.2.4 differ from those for Figure 4.2.3 only in the ordering of the nodes. For Figure 4.2.4 we select for every $n \geq 1$ the nodes

$$
z_{k}:=2 \cos (\pi k / n), \quad 0 \leq k \leq n \text {. }
$$

Example 4.3. We consider the solution of primal CV systems (1.5) by Algorithm 2 using single precision arithmetic. Let $\mathbf{x}^{*} \in \mathbb{C}^{n+1}$ denote the computed solution, and define the residual error

$$
\mathbf{r}^{\prime}:=V_{\rho} \mathbf{x}^{*}-\mathbf{g}
$$

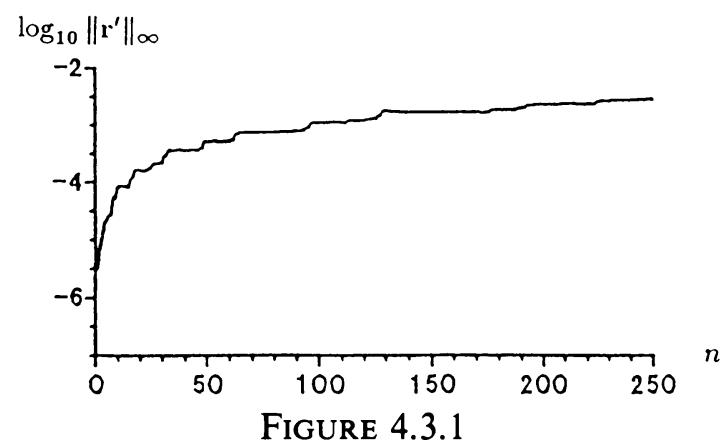

Growth of $\left\|\mathbf{r}^{\prime}\right\|_{\infty}$ with $n$ for $\rho=0.8$ and nodes (1.9) 
Figure 4.3.1 shows $\left\|\mathbf{r}^{\prime}\right\|_{\infty}$ for $\rho=0.8$ and the nodes (1.9). The right-hand side $\mathbf{g}=\left[g_{j}\right]_{j=0}^{n}$ is given by $g_{j}:=\exp \left(z_{j}\right)$.

Numerous numerical experiments indicate that the residual error (4.4) often is somewhat larger than the error (4.1) for identical matrices $V_{\rho}$ and right-hand sides. Further computed examples can be found in [16].

\section{ConClusions}

Fast progressive algorithms are derived for the solution of CV systems, and in $\S \S 3$ and 4 these algorithms are demonstrated to be fairly insensitive to perturbations for suitably distributed and ordered nodes.

\section{ACKNOWLEDGMENT}

We would like to thank Åke Björck and Arnold Schönhage for helpful discussions.

Addendum. Figure 4.1.1 inspired A. Córdova, W. Gautschi, and S. Ruscheweyh to completely describe the spectrum and eigenvectors of $V_{0} V_{0}^{H}$ in the paper Vandermonde matrices on the circle: spectral properties and conditioning, Numer. Math. 57 (1990), 577-591. In particular, they show that $\kappa_{2}\left(V_{0}\right)=O\left(n^{1 / 2}\right)$ for $V_{0} \in \mathbb{C}^{(n+1) \times(n+1)}$ defined by the nodes (1.9) with $\rho=0$. A survey of condition number bounds for Vandermonde matrices can be found in the paper How (un)stable are Vandermonde systems? by W. Gautschi, in Asymptotic and Computational Analysis (R. Wong, ed.), Lecture Notes in Pure and Appl. Math., vol. 124, Dekker, New York, 1990, pp. 193-210.

\section{BIBLIOGRAPHY}

1. A. Björck and V. Pereyra, Solution of Vandermonde systems of equations, Math. Comp. 24 (1970), 893-903.

2. P. J. Davis, Interpolation and approximation, Dover, New York, 1975.

3. J. J. Dongarra, C. B. Moler, J. R. Bunch, and G. W. Stewart, Linpack users' guide, SIAM, Philadelphia, PA, 1979.

4. S. W. Ellacott, Computation of Faber series with application to numerical polynomial approximation in the complex plane, Math. Comp. 40 (1984), 575-587.

5. Engineering and scientific subroutine library, IBM, 1987.

6. B. Fischer and L. Reichel, A stable Richardson iteration method for complex linear systems, Numer. Math. 54 (1988), 225-242. 7. $\frac{1}{278}$. Newton interpolation in Fejér and Chebyshev points, Math. Comp. 53 (1989), 265-

8. W. Gautschi and G. Inglese, Lower bounds for the condition number of Vandermonde matrices, Numer. Math. 52 (1988), 241-250.

9. W. Gautschi, The condition of Vandermonde-like matrices involving orthogonal polynomials, Linear Algebra Appl. 52/53 (1983), 293-300.

10. _ The condition of polynomials in power form, Math. Comp. 33 (1979), 343-352.

11. __ Optimally conditioned Vandermonde matrices, Numer. Math. 24 (1975), 1-12. 
12. G. H. Golub and C. F. Van Loan, Matrix computations, 2nd ed., Johns Hopkins Univ. Press, Baltimore, MD, 1989.

13. N. J. Higham, Fast solution of Vandermonde-like systems involving orthogonal polynomials, IMA J. Numer. Anal. 8 (1988), 473-486.

14. _ Stability analysis of algorithms for solving confluent Vandermonde-like systems, SIAM J. Matrix Anal. Appl. 11 (1990), 23-41.

15. E. Hlawka, The theory of uniform distribution, AB Academic Publishers, Berkhamsted, 1984.

16. L. Reichel and G. Opfer, Chebyshev-Vandermonde systems, BSC Report 88/48, Bergen Scientific Centre, Bergen, Norway, 1988.

17. V. I. Smirnov and N. A. Lebedev, Functions of a complex variable, Iliffe Books, London, 1968.

18. W. P. Tang and G. H. Golub, The block decompositions of a Vandermonde matrix and its applications, BIT 21 (1981), 505-517.

Department of Mathematics, University of Kentucky, Lexington, Kentucky 40506

INSTitute of APPLiEd MATHEMATICS, UNiversity of HAMBURG, BUNDESSTRASSE 55, D-2000 HAMBURG 13, GeRMANY 